\title{
On Generalized p-Mersenne Numbers
}

\author{
Yüksel Soykan
}

Department of Mathematics, Art and Science Faculty,

Zonguldak Bülent Ecevit University, 67100, Zonguldak, Turkey

e-mail: yuksel_soykan@hotmail.com

\begin{abstract}
In this paper, we introduce the generalized p-Mersenne sequence and deal with, in detail, two special cases, namely, p-Mersenne and p-Mersenne-Lucas sequences. We present Binet's formulas, generating functions, Simson formulas, and the summation formulas for these sequences. Moreover, we give some identities and matrices related with these sequences.
\end{abstract}

\section{Introduction}

A Mersenne number, denoted by $M_{n}$, and a Mersenne-Lucas number, denoted by $H_{n}$, are numbers of the form $M_{n}=2^{n}-1$ and $H_{n}=2^{n}+1$, respectively. The Mersenne sequence $\left\{M_{n}\right\}_{n \geq 0}$ and the Mersenne-Lucas sequence $\left\{H_{n}\right\}_{n \geq 0}$ can also be defined recursively by

$$
M_{n}=3 M_{n-1}-2 M_{n-2}
$$

with initial conditions $M_{0}=0, M_{1}=1$ and

$$
H_{n}=3 H_{n-1}-2 H_{n-2}
$$

with initial conditions $H_{0}=2, H_{1}=3$, respectively.

$\left\{M_{n}\right\}_{n \geq 0}$ is the sequence $A 000225$ in the OEIS [21, whereas $\left\{H_{n}\right\}_{n \geq 0}$ is the id-number $A 000051$ in OEIS. Note that Mersenne-Lucas numbers are also

Received: October 1, 2021; Accepted: November 6, 2021

2020 Mathematics Subject Classification: 11B37, 11B39, $11 B 83$.

Keywords and phrases: Mersenne numbers, Mersenne-Lucas numbers, p-Mersenne numbers, p-Mersenne-Lucas numbers, generalized Fibonacci numbers. 
called as Fermat numbers. In fact, there are two definitions of the Fermat numbers. The less common is a number of the form $2^{n}+1$, the first few of which are $2,3,5,9,17,33, \ldots$ (OEIS A000051). The much more commonly encountered Fermat numbers are a special case, given by the binomial number of the form $F_{n}=2^{2^{n}}+1$. The first few for $n=0,1,2, \ldots$ are $3,5,17,257,65537,4294967297, \ldots$ (OEIS A000215).

Mersenne sequence has been studied by many authors and more detail can be found in the extensive literature dedicated to this sequence, see for example,

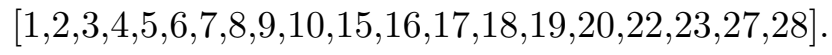

Generalizations of Mersenne numbers can be obtained in various ways (see for example $\left.\begin{array}{ll|l|l|l|l|l|l}5 & 10 & 17 & 22\end{array}\right)$. Our generalizations of Mersenne numbers in Section 2 are not Mersenne numbers in the sense of 10,22 and are Mersenne numbers in the sense of 23 which is given as: a generalized Mersenne sequence $\left\{W_{n}\right\}_{n \geq 0}=$ $\left\{W_{n}\left(W_{0}, W_{1}\right)\right\}_{n \geq 0}$ is defined by the second-order recurrence relation

$$
W_{n}=3 W_{n-1}-2 W_{n-2}
$$

with the initial values $W_{0}=c_{0}, W_{1}=c_{1}$ not all being zero. For more information on generalized Mersenne numbers, see Soykan 23.

The purpose of this article is to generalize and investigate these interesting sequence of numbers (Mersenne numbers). First, we recall some properties of Fibonacci numbers and its generalizations, namely generalized Fibonacci numbers.

The Fibonacci numbers and their generalizations have many interesting properties and applications to almost every field such as architecture, nature, art, physics and engineering. The sequence of Fibonacci numbers $\left\{F_{n}\right\}_{n \geq 0}$ is defined by

$$
F_{n}=F_{n-1}+F_{n-2}, \quad n \geq 2, \quad F_{0}=0, \quad F_{1}=1 .
$$

The generalization of Fibonacci sequence leads to several nice and interesting sequences. The generalized Fibonacci sequence (or generalized $(r, s)$-sequence or Horadam sequence or 2-step Fibonacci sequence) $\left\{W_{n}\left(W_{0}, W_{1} ; r, s\right)\right\}_{n \geq 0}$ (or shortly $\left\{W_{n}\right\}_{n \geq 0}$ ) is defined (by Horadam 12) as follows:

$$
W_{n}=r W_{n-1}+s W_{n-2}, \quad W_{0}=a, W_{1}=b, \quad n \geq 2
$$


where $W_{0}, W_{1}$ are arbitrary complex (or real) numbers and $r, s$ are real numbers, see also Horadam 11/13 14 and Soykan 25].

For some specific values of $a, b, r$ and $s$, it is worth presenting these special Horadam numbers in a table as a specific name. In literature, for example, the following names and notations (see Table 1) are used for the special cases of $r, s$ and initial values.

Table 1: A few special case of generalized Fibonacci sequences.

\begin{tabular}{cccc}
\hline Name of sequence & $W_{n}(a, b ; r, s)$ & Binet Formula & OEIS[21] \\
\cline { 1 - 2 } Fibonacci & $W_{n}(0,1 ; 1,1)=F_{n}$ & $\frac{\left(\frac{1+\sqrt{5}}{2}\right)^{n}-\left(\frac{1-\sqrt{5}}{2}\right)^{n}}{\text { Lucas }}$ & A000045 \\
Pell & $W_{n}(2,1 ; 1,1)=L_{n}$ & $\left(\frac{1+\sqrt{5}}{2}\right)^{n}+\left(\frac{1-\sqrt{5}}{2}\right)^{n}$ & A000032 \\
Pell-Lucas & $W_{n}(0,1 ; 2,1)=P_{n}$ & $\frac{(1+\sqrt{2})^{n}-(1-\sqrt{2})^{n}}{2 \sqrt{2}}$ & A000129 \\
Jacobsthal & $W_{n}(2,2 ; 2,1)=Q_{n}$ & $(1+\sqrt{2})^{n}+(1-\sqrt{2})^{n}$ & A002203 \\
Jacobsthal-Lucas & $W_{n}(0,1 ; 1,2)=J_{n}$ & $\frac{2^{n}-(-1)^{n}}{3}$ & A001045 \\
\hline
\end{tabular}

Here, OEIS stands for On-line Encyclopedia of Integer Sequences.

The sequence $\left\{W_{n}\right\}_{n \geq 0}$ can be extended to negative subscripts by defining

$$
W_{-n}=-\frac{r}{s} W_{-(n-1)}+\frac{1}{s} W_{-(n-2)}
$$

for $n=1,2,3, \ldots$ when $s \neq 0$. Therefore, recurrence 1.2 holds for all integer $n$.

Now we define two special cases of the sequence $\left\{W_{n}\right\}$. $(r, s)$ sequence $\left\{G_{n}(0,1 ; r, s)\right\}_{n \geq 0}$ and Lucas $(r, s)$ sequence $\left\{H_{n}(2, r ; r, s)\right\}_{n \geq 0}$ are defined, respectively, by the second-order recurrence relations

$$
\begin{array}{ll}
G_{n+2}=r G_{n+1}+s G_{n}, & G_{0}=0, G_{1}=1, \\
H_{n+2}=r H_{n+1}+s H_{n}, & H_{0}=2, H_{1}=r .
\end{array}
$$

The sequences $\left\{G_{n}\right\}_{n \geq 0},\left\{H_{n}\right\}_{n \geq 0}$ and $\left\{E_{n}\right\}_{n \geq 0}$ can be extended to negative 
subscripts by defining

$$
\begin{aligned}
& G_{-n}=-\frac{r}{s} G_{-(n-1)}+\frac{1}{s} G_{-(n-2)}, \\
& H_{-n}=-\frac{r}{s} H_{-(n-1)}+\frac{1}{s} H_{-(n-2)},
\end{aligned}
$$

for $n=1,2,3, \ldots$ respectively. Therefore, recurrences 1.3 - 1.4 hold for all integer $n$.

Some special cases of $(r, s)$ sequence $\left\{G_{n}(0,1 ; r, s)\right\}_{n \geq 0}$ and Lucas $(r, s)$ sequence $\left\{H_{n}(2, r ; r, s)\right\}_{n \geq 0}$ are as follows:

1. $G_{n}(0,1 ; 1,1)=F_{n}$, Fibonacci sequence,

2. $H_{n}(2,1 ; 1,1)=L_{n}$, Lucas sequence,

3. $G_{n}(0,1 ; 2,1)=P_{n}$, Pell sequence,

4. $H_{n}(2,2 ; 2,1)=Q_{n}$, Pell-Lucas sequence,

5. $G_{n}(0,1 ; 1,2)=J_{n}$, Jacobsthal sequence,

6. $H_{n}(2,1 ; 1,2)=j_{n}$, Jacobsthal-Lucas sequence.

The following theorem shows that the generalized Fibonacci sequence $W_{n}$ at negative indices can be expressed by the sequence itself at positive indices.

Theorem 1. For $n \in \mathbb{Z}$, for the generalized Fibonacci sequence (or generalized $(r, s)$-sequence or Horadam sequence or 2-step Fibonacci sequence) we have the following:

(a)

$$
\begin{aligned}
W_{-n} & =(-1)^{-n-1} s^{-n}\left(W_{n}-H_{n} W_{0}\right) \\
& =(-1)^{n+1} s^{-n}\left(W_{n}-H_{n} W_{0}\right) .
\end{aligned}
$$

(b)

$$
W_{-n}=\frac{(-1)^{n+1} s^{-n}}{-W_{1}^{2}+s W_{0}^{2}+r W_{0} W_{1}}\left(\left(2 W_{1}-r W_{0}\right) W_{0} W_{n+1}-\left(W_{1}^{2}+s W_{0}^{2}\right) W_{n}\right) .
$$


Proof. For the proof, see Soykan 26, Theorem 3.2 and Theorem 3.3].

The following theorem presents sum formulas of generalized $(r, s)$ numbers (generalized Fibonacci numbers).

Theorem 2. Let $x$ be a real (or complex) number. For all integers $m$ and $j$, for generalized $(r, s)$ numbers (generalized Fibonacci numbers), we have the following sum formulas:

(a) If $(-s)^{m} x^{2}-x H_{m}+1 \neq 0$, then

$$
\sum_{k=0}^{n} x^{k} W_{m k+j}=\frac{\left((-s)^{m} x-H_{m}\right) x^{n+1} W_{m n+j}+(-s)^{m} x^{n+1} W_{m n+j-m}}{+W_{j}-(-s)^{m} x W_{j-m}} .
$$

(b) If $(-s)^{m} x^{2}-x H_{m}+1=u(x-a)(x-b)=0$ for some $u, a, b \in \mathbb{C}$ with $u \neq 0$ and $a \neq b$, i.e., $x=a$ or $x=b$, then

$$
\sum_{k=0}^{n} x^{k} W_{m k+j}=\frac{\begin{array}{c}
\left(x(n+2)(-s)^{m}-(n+1) H_{m}\right) x^{n} W_{j+m n} \\
+(-s)^{m}(n+1) x^{n} W_{m n+j-m}-(-s)^{m} W_{j-m}
\end{array}}{2(-s)^{m} x-H_{m}} .
$$

(c) If $(-s)^{m} x^{2}-x H_{m}+1=u(x-c)^{2}=0$ for some $u, c \in \mathbb{C}$ with $u \neq 0$, i.e., $x=c$, then

$$
\sum_{k=0}^{n} x^{k} W_{m k+j}=\frac{\begin{array}{c}
(n+1)\left((-s)^{m}(n+2) x^{n}-n x^{n-1} H_{m}\right) W_{m n+j} \\
+n(n+1)(-s)^{m} x^{n-1} W_{m n+j-m}
\end{array}}{2(-s)^{m}} .
$$

Proof. It is given in Soykan 26, Theorem 4.1].

Note that 1.5 can be written in the following form

$$
\sum_{k=1}^{n} x^{k} W_{m k+j}=\frac{\begin{array}{c}
\left((-s)^{m} x-H_{m}\right) x^{n+1} W_{m n+j}+(-s)^{m} x^{n+1} W_{m n+j-m} \\
+x\left(H_{m}-(-s)^{m} x\right) W_{j}-(-s)^{m} x W_{j-m}
\end{array}}{(-s)^{m} x^{2}-x H_{m}+1} .
$$

We give the ordinary generating function $\sum_{n=0}^{\infty} W_{n} x^{n}$ of the sequence $\left\{W_{n}\right\}$. 
Lemma 3. Suppose that $f_{W_{n}}(x)=\sum_{n=0}^{\infty} W_{n} x^{n}$ is the ordinary generating function of the generalized Fibonacci sequence $\left\{W_{n}\right\}_{n \geq 0}$. Then, $\sum_{n=0}^{\infty} W_{n} x^{n}$ is given by

$$
\sum_{n=0}^{\infty} W_{n} x^{n}=\frac{W_{0}+\left(W_{1}-r W_{0}\right) x}{1-r x-s x^{2}} .
$$

Proof. For a proof see, 25, Lemma 1.1].

\subsection{Binet's Formula for the Distinct Roots Case and Single Root Case}

Let $\alpha$ and $\beta$ be two roots of the quadratic equation

$$
x^{2}-r x-s=0,
$$

of which the left-hand side is called the characteristic polynomial (or the characteristic equation) of the recurrence relation 1.2 . The following theorem presents the Binet's formula of the sequence $W_{n}$.

Theorem 4. The general term of the sequence $W_{n}$ can be presented by the following Binet formula:

$$
\begin{aligned}
W_{n} & =\left\{\begin{array}{lc}
\frac{W_{1}-\beta W_{0}}{\alpha-\beta} \alpha^{n}-\frac{W_{1}-\alpha W_{0}}{\alpha-\beta} \beta^{n} & , \quad \text { if } \alpha \neq \beta \text { (Distinct Roots Case) } \\
\left(n W_{1}-\alpha(n-1) W_{0}\right) \alpha^{n-1} & , \quad \text { if } \alpha=\beta \text { (Single Root Case) }
\end{array}\right. \\
& =\left\{\begin{array}{lc}
\frac{W_{1}-\beta W_{0}}{\alpha-\beta} \alpha^{n}-\frac{W_{1}-\alpha W_{0}}{\alpha-\beta} \beta^{n} & , \text { if } \alpha \neq \beta \text { (Distinct Roots Case) } \\
\left(n W_{1}-\frac{r}{2}(n-1) W_{0}\right)\left(\frac{r}{2}\right)^{n-1} & , \quad \text { if } \alpha=\beta \text { (Single Root Case) }
\end{array} .\right.
\end{aligned}
$$

Proof. For a proof, see Soykan 25 and 26.

The roots of characteristic equation are

$$
\alpha=\frac{r+\sqrt{\Delta}}{2}, \beta=\frac{r-\sqrt{\Delta}}{2} .
$$

where

$$
\Delta=r^{2}+4 s
$$


and the followings hold

$$
\begin{aligned}
\alpha+\beta & =r \\
\alpha \beta & =-s, \\
(\alpha-\beta)^{2} & =(\alpha+\beta)^{2}-4 \alpha \beta=r^{2}+4 s .
\end{aligned}
$$

If $\Delta=r^{2}+4 s \neq 0$, then $\alpha \neq \beta$, i.e., there are distinct roots of the quadratic equation (1.7) and if $\Delta=r^{2}+4 s=0$, then $\alpha=\beta$, i.e., there is a single root of the quadratic equation (1.7).

In the case $r^{2}+4 s \neq 0$ so that $\alpha \neq \beta$, for all integers $n,(r, s)$ and Lucas $(r, s)$ numbers (using initial conditions in Theorem 4 4 can be expressed using Binet's formulas as

$$
\begin{aligned}
& G_{n}=\frac{\alpha^{n}-\beta^{n}}{\alpha-\beta}, \\
& H_{n}=\alpha^{n}+\beta^{n},
\end{aligned}
$$

respectively. In the case $r^{2}+4 s=0$ so that $\alpha=\beta$, for all integers $n,(r, s)$ and Lucas $(r, s)$ numbers (using initial conditions in Theorem 4) can be expressed using Binet's formulas as

$$
\begin{aligned}
& G_{n}=n \alpha^{n-1}, \\
& H_{n}=2 \alpha^{n},
\end{aligned}
$$

respectively.

\section{Generalized p-Mersenne Sequence}

In this paper, we consider the case $r=p, s=-(p-1)$ where $p \in \mathbb{R}, p \neq 1$ (so throughout the paper we assume that $p \neq 1$ unless otherwise stated). A generalized p-Mersenne sequence $\left\{W_{n}\right\}_{n \geq 0}=\left\{W_{n}\left(W_{0}, W_{1}\right)\right\}_{n \geq 0}$ is defined by the second-order recurrence relation

$$
W_{n}=p W_{n-1}-(p-1) W_{n-2}
$$


with the initial values $W_{0}=c_{0}, W_{1}=c_{1}$ not all being zero.

The sequence $\left\{W_{n}\right\}_{n \geq 0}$ can be extended to negative subscripts by defining

$$
W_{-n}=-\frac{p}{(p-1)} W_{-(n-1)}-\frac{1}{(p-1)} W_{-(n-2)}
$$

for $n=1,2,3, \ldots$. Therefore, recurrence (2.1) holds for all integer $n$.

By Theorem 4, the Binet formula of generalized p-Mersenne numbers can be written as

$$
W_{n}=\left\{\begin{array}{cc}
\frac{W_{1}-\beta W_{0}}{\alpha-\beta} \alpha^{n}-\frac{W_{1}-\alpha W_{0}}{\alpha-\beta} \beta^{n} & , \quad \text { if } \alpha \neq \beta \text { (Distinct Roots Case) } \\
\left(n W_{1}-\frac{r}{2}(n-1) W_{0}\right)\left(\frac{r}{2}\right)^{n-1} & , \quad \text { if } \alpha=\beta \text { (Single Root Case) }
\end{array}\right.
$$

where $\alpha$ and $\beta$ are the roots of the quadratic equation $x^{2}-p x+(p-1)=0$. Moreover

$$
\begin{aligned}
& \alpha=p-1 \\
& \beta=1
\end{aligned}
$$

Note that

$$
\begin{aligned}
\alpha+\beta & =p, \\
\alpha \beta & =p-1, \\
\alpha-\beta & =p-2 .
\end{aligned}
$$

So

$$
W_{n}=\left\{\begin{array}{cc}
\frac{W_{1}-W_{0}}{p-2}(p-1)^{n}-\frac{W_{1}-(p-1) W_{0}}{p-2} & , \text { if } p \neq 2 \\
n W_{1}-(n-1) W_{0} & , \text { if } p=2
\end{array}\right.
$$

For the case $p \in \mathbb{R}, p \neq 1$, the first few generalized p-Mersenne numbers with positive subscript and negative subscript are given in the following Table 2.

Table 2: A few generalized p-Mersenne numbers.

\begin{tabular}{lcc}
\hline$n$ & $W_{n}$ & $W_{-n}$ \\
\hline 0 & $W_{0}$ & $W_{0}$ \\
1 & $W_{1}$ & $\frac{1}{p-1}\left(-W_{1}+p W_{0}\right)$ \\
2 & $p W_{1}-(p-1) W_{0}$ & $\frac{1}{(p-1)^{2}}\left(-p W_{1}+\left(p^{2}-p+1\right) W_{0}\right)$ \\
3 & $\left(p^{2}-p+1\right) W_{1}-p(p-1) W_{0}$ & $\frac{1}{(p-1)^{3}}\left(-\left(p^{2}-p+1\right) W_{1}+p\left(p^{2}-2 p+2\right) W_{0}\right)$ \\
4 & $p\left(p^{2}-2 p+2\right) W_{1}-(p-1)\left(p^{2}-p+1\right) W_{0}$ & $\frac{1}{(p-1)^{4}}\left(-p\left(p^{2}-2 p+2\right) W_{1}+\left(p^{4}-3 p^{3}+4 p^{2}-2 p+1\right) W_{0}\right)$ \\
\hline
\end{tabular}


For the case $p=2$, the first few generalized 2-Mersenne numbers with positive subscript and negative subscript are given in the following Table 3.

Table 3: A few generalized 2-Mersenne numbers.

\begin{tabular}{ccc}
\hline$n$ & $W_{n}$ & $W_{-n}$ \\
\hline 0 & $W_{0}$ & $W_{0}$ \\
1 & $W_{1}$ & $W_{-1}=2 W_{0}-W_{1}$ \\
2 & $W_{2}=2 W_{1}-W_{0}$ & $W_{-2}=3 W_{0}-2 W_{1}$ \\
3 & $W_{3}=3 W_{1}-2 W_{0}$ & $W_{-3}=4 W_{0}-3 W_{1}$ \\
4 & $W_{4}=4 W_{1}-3 W_{0}$ & $W_{-4}=5 W_{0}-4 W_{1}$ \\
5 & $W_{5}=5 W_{1}-4 W_{0}$ & $W_{-5}=6 W_{0}-5 W_{1}$ \\
6 & $W_{6}=6 W_{1}-5 W_{0}$ & $W_{-6}=7 W_{0}-6 W_{1}$ \\
7 & $W_{7}=7 W_{1}-6 W_{0}$ & $W_{-7}=8 W_{0}-7 W_{1}$ \\
8 & $W_{8}=8 W_{1}-7 W_{0}$ & $W_{-8}=9 W_{0}-8 W_{1}$ \\
\hline & &
\end{tabular}

Now we define two special cases of the sequence $\left\{W_{n}\right\}$. p-Mersenne sequence $\left\{M_{n}\right\}_{n \geq 0}$ and p-Mersenne-Lucas sequence $\left\{H_{n}\right\}_{n \geq 0}$ are defined, respectively, by the second-order recurrence relations

$$
\begin{array}{rlr}
M_{n} & =p M_{n-1}-(p-1) M_{n-2}, & M_{0}=0, M_{1}=1, \\
H_{n} & =p H_{n-1}-(p-1) H_{n-2}, & H_{0}=2, H_{1}=p,
\end{array}
$$

The sequences $\left\{M_{n}\right\}_{n \geq 0}$ and $\left\{H_{n}\right\}_{n \geq 0}$ can be extended to negative subscripts by defining

$$
\begin{aligned}
& M_{-n}=-\frac{p}{(p-1)} M_{-(n-1)}-\frac{1}{(p-1)} M_{-(n-2)}, \\
& H_{-n}=-\frac{p}{(p-1)} H_{-(n-1)}-\frac{1}{(p-1)} H_{-(n-2)},
\end{aligned}
$$

for $n=1,2,3, \ldots$ respectively. Therefore, recurrences 2.3 - 2.4 hold for all integer $n$.

If $p=3$, then 3-Mersenne sequence $\left\{M_{n}\right\}$ is the well known Mersenne sequence and 3-Mersenne-Lucas sequence $\left\{H_{n}\right\}$ is the well known Mersenne-Lucas sequence. If $p=2$, then 2-Mersenne sequence $\left\{M_{n}\right\}_{n \geq 0}$ is the sequence $A 001477$ in the OEIS 
21, whereas 2-Mersenne-Lucas sequence $\left\{H_{n}\right\}_{n \geq 0}$ is the id-number $A 007395$ in OEIS.

Next, for the case $p \in \mathbb{R}, p \neq 1$, we present the first few values of the p-Mersenne and p-Mersenne-Lucas numbers with positive and negative subscripts:

Table 4: The first few values of the special second-order numbers with positive and negative subscripts.

\begin{tabular}{cccccc}
\hline$n$ & 0 & 1 & 2 & 3 & 4 \\
\hline$M_{n}$ & 0 & 1 & $p$ & $p^{2}-p+1$ & $p\left(p^{2}-2 p+2\right)$ \\
$M_{-n}$ & $\cdots$ & $-\frac{1}{p-1}$ & $-\frac{p}{(p-1)^{2}}$ & $-\frac{1}{(p-1)^{3}}\left(p^{2}-p+1\right)$ & $-\frac{p}{(p-1)^{4}}\left(p^{2}-2 p+2\right)$ \\
$H_{n}$ & 2 & $p$ & $p^{2}-2 p+2$ & $p\left(p^{2}-3 p+3\right)$ & $p^{4}-4 p^{3}+6 p^{2}-4 p+2$ \\
$H_{-n}$ & $\cdots$ & $\frac{p}{p-1}$ & $\frac{1}{(p-1)^{2}}\left(p^{2}-2 p+2\right)$ & $\frac{p}{(p-1)^{3}}\left(p^{2}-3 p+3\right)$ & $\frac{1}{(p-1)^{4}}\left(p^{4}-4 p^{3}+6 p^{2}-4 p+2\right)$ \\
\hline
\end{tabular}

Now, for the case $p=2$, we present the first few values of the 2-Mersenne and 2-Mersenne-Lucas numbers with positive and negative subscripts:

Table 5: The first few values of the special second-order numbers with positive and negative subscripts.

\begin{tabular}{ccccccccccccccccc}
\hline$n$ & 0 & 1 & 2 & 3 & 4 & 5 & 6 & 7 & 8 & 9 & 10 & 11 & 12 & 13 & 14 & 15 \\
\hline$M_{n}$ & 0 & 1 & 2 & 3 & 4 & 5 & 6 & 7 & 8 & 9 & 10 & 11 & 12 & 13 & 14 & 15 \\
$M_{-n}$ & $\ldots$ & -1 & -2 & -3 & -4 & -5 & -6 & -7 & -8 & -9 & -10 & -11 & -12 & -13 & -14 & -15 \\
$H_{n}$ & 2 & 2 & 2 & 2 & 2 & 2 & 2 & 2 & 2 & 2 & 2 & 2 & 2 & 2 & 2 & 2 \\
$H_{-n}$ & $\cdots$ & 2 & 2 & 2 & 2 & 2 & 2 & 2 & 2 & 2 & 2 & 2 & 2 & 2 & 2 & 2 \\
\hline
\end{tabular}

For all integers $n$, p-Mersenne and p-Mersenne-Lucas (using initial conditions in Theorem 4) can be expressed using Binet's formulas as

$$
M_{n}=\left\{\begin{array}{cl}
\frac{1}{p-2}(p-1)^{n}-\frac{1}{p-2} & , \text { if } p \neq 2 \\
n & , \text { if } p=2
\end{array}\right.
$$

and

$$
H_{n}=\left\{\begin{array}{cl}
(p-1)^{n}+1 & , \text { if } p \neq 2 \\
2 & , \text { if } p=2
\end{array}=(p-1)^{n}+1\right.
$$

respectively. 
Next, we give the ordinary generating function $\sum_{n=0}^{\infty} W_{n} x^{n}$ of the sequence $\left\{W_{n}\right\}$.

Lemma 5. Suppose that $f_{W_{n}}(x)=\sum_{n=0}^{\infty} W_{n} x^{n}$ is the ordinary generating function of the generalized p-Mersenne sequence $\left\{W_{n}\right\}_{n \geq 0}$. Then, $\sum_{n=0}^{\infty} W_{n} x^{n}$ is given by

$$
\sum_{n=0}^{\infty} W_{n} x^{n}=\frac{W_{0}+\left(W_{1}-p W_{0}\right) x}{1-p x+(p-1) x^{2}} .
$$

Proof. In Lemma 3, take $r=p, s=-(p-1)$.

The previous Lemma gives the following results as particular examples.

Corollary 6. Generated functions of p-Mersenne and p-Mersenne-Lucas numbers are

$$
\begin{aligned}
\sum_{n=0}^{\infty} M_{n} x^{n} & =\frac{x}{1-p x+(p-1) x^{2}}, \\
\sum_{n=0}^{\infty} H_{n} x^{n} & =\frac{2-p x}{1-p x+(p-1) x^{2}},
\end{aligned}
$$

respectively.

Proof. In Lemma 5, take $W_{n}=M_{n}$ with $M_{0}=0, M_{1}=1$ and $W_{n}=H_{n}$ with $H_{0}=2, H_{1}=p$, respectively.

\section{Simson's Formulas}

There is a well-known Simson's Identity (formula) for Fibonacci sequence $\left\{F_{n}\right\}$, namely,

$$
F_{n+1} F_{n-1}-F_{n}^{2}=(-1)^{n}
$$

which was derived first by R. Simson in 1753 and it is now called as Cassini's Identity (formula) as well. This can be written in the form

$$
\begin{array}{cc}
F_{n+1} & F_{n} \\
F_{n} & F_{n-1}
\end{array} \mid=(-1)^{n} .
$$


The following theorem gives generalization of this result to the generalized p-Mersenne sequence $\left\{W_{n}\right\}_{n \geq 0}$.

Theorem 7 (Simson's Formula of Generalized p-Mersenne Numbers). For all integers $n$, we have

$$
\left|\begin{array}{cc}
W_{n+1} & W_{n} \\
W_{n} & W_{n-1}
\end{array}\right|=(p-1)^{n-1}\left(-W_{1}^{2}-(p-1) W_{0}^{2}+p W_{1} W_{0}\right) .
$$

Proof. For a proof of Eq. (3.1), see Soykan [24, just take $s=-(p-1)$.

The previous theorem gives the following results as particular examples.

Corollary 8. For all integers n, p-Mersenne and p-Mersenne-Lucas numbers are given as

$$
\begin{aligned}
\left|\begin{array}{cc}
M_{n+1} & M_{n} \\
M_{n} & M_{n-1}
\end{array}\right| & =-(p-1)^{n-1}, \\
\left|\begin{array}{cc}
H_{n+1} & H_{n} \\
H_{n} & H_{n-1}
\end{array}\right| & =(p-1)^{n-1}\left(p^{2}-4 p+4\right),
\end{aligned}
$$

respectively.

\section{Some Identities}

In this section, we obtain some identities of generalized p-Mersenne, p-Mersenne and p-Mersenne-Lucas numbers. First, we can give a few basic relations between $\left\{W_{n}\right\}$ and $\left\{M_{n}\right\}$.

Lemma 9. The following equalities are true:

(a) $(p-1)^{3} W_{n}=\left(-\left(p^{2}-p+1\right) W_{1}+p\left(p^{2}-2 p+2\right) W_{0}\right) M_{n+4}+\left(p\left(p^{2}-2 p+\right.\right.$ 2) $\left.W_{1}-\left(p^{4}-3 p^{3}+4 p^{2}-2 p+1\right) W_{0}\right) M_{n+3}$.

(b) $(p-1)^{2} W_{n}=\left(-p W_{1}+\left(p^{2}-p+1\right) W_{0}\right) M_{n+3}+\left(\left(p^{2}-p+1\right) W_{1}-p\left(p^{2}-2 p+\right.\right.$ 2) $\left.W_{0}\right) M_{n+2}$. 
(c) $(p-1) W_{n}=\left(-W_{1}+p W_{0}\right) M_{n+2}-\left(-p W_{1}+\left(p^{2}-p+1\right) W_{0}\right) M_{n+1}$.

(d) $W_{n}=W_{0} M_{n+1}+\left(W_{1}-p W_{0}\right) M_{n}$.

(e) $W_{n}=W_{1} M_{n}-(p-1) W_{0} M_{n-1}$.

(f) $(p-1)^{3}\left(W_{0}-W_{1}\right)\left(-W_{1}+(p-1) W_{0}\right) M_{n}=\left(-\left(p^{2}-p+1\right) W_{1}+\right.$ $\left.p(p-1) W_{0}\right) W_{n+4}+\left(p\left(p^{2}-2 p+2\right) W_{1}-(p-1)\left(p^{2}-p+1\right) W_{0}\right) W_{n+3}$.

(g) $(p-1)^{2}\left(W_{0}-W_{1}\right)\left(-W_{1}+(p-1) W_{0}\right) M_{n}=\left(-p W_{1}+(p-1) W_{0}\right) W_{n+3}+$ $\left(\left(p^{2}-p+1\right) W_{1}-p(p-1) W_{0}\right) W_{n+2}$.

(h) $(p-1)\left(W_{0}-W_{1}\right)\left(-W_{1}+(p-1) W_{0}\right) M_{n}=-W_{1} W_{n+2}+\left(p W_{1}+(1-\right.$ p) $\left.W_{0}\right) W_{n+1}$.

(i) $\left(W_{0}-W_{1}\right)\left(-W_{1}+(p-1) W_{0}\right) M_{n}=-W_{0} W_{n+1}+W_{1} W_{n}$.

(j) $\left(W_{0}-W_{1}\right)\left(-W_{1}+(p-1) W_{0}\right) M_{n}=\left(W_{1}-p W_{0}\right) W_{n}+(p-1) W_{0} W_{n-1}$.

Proof. Note that all the identities hold for all integers $n$. We prove (a). To show (a), writing

$$
W_{n}=a \times M_{n+4}+b \times M_{n+3}
$$

and solving the system of equations

$$
\begin{aligned}
& W_{0}=a \times M_{4}+b \times M_{3} \\
& W_{1}=a \times M_{5}+b \times M_{4}
\end{aligned}
$$

we find that $a=\frac{\left(-\left(p^{2}-p+1\right) W_{1}+p\left(p^{2}-2 p+2\right) W_{0}\right)}{(p-1)^{3}}, \quad b=$ $\frac{\left(p\left(p^{2}-2 p+2\right) W_{1}-\left(p^{4}-3 p^{3}+4 p^{2}-2 p+1\right) W_{0}\right)}{(p-1)^{3}}$. The other equalities can be proved similarly.

Note that all the identities in the above Lemma can be proved by induction as well.

Next, we present a few basic relations between $\left\{H_{n}\right\}$ and $\left\{W_{n}\right\}$.

Lemma 10. The following equalities are true: 
(a) $(p-2)^{2}(p-1)^{3} W_{n}=-\left(-p\left(p^{2}-3 p+3\right) W_{1}+\left(p^{4}-4 p^{3}+6 p^{2}-4 p+\right.\right.$ 2) $\left.W_{0}\right) H_{n+4}+\left(-\left(p^{4}-4 p^{3}+6 p^{2}-4 p+2\right) W_{1}+p\left(p^{4}-5 p^{3}+10 p^{2}-10 p+\right.\right.$ 5) $\left.W_{0}\right) H_{n+3}$.

(b) $(p-1)^{2}(p-2)^{2} W_{n}=\left(\left(p^{2}-2 p+2\right) W_{1}-p\left(p^{2}-3 p+3\right) W_{0}\right) H_{n+3}+\left(-p\left(p^{2}-\right.\right.$ $\left.3 p+3) W_{1}+\left(p^{4}-4 p^{3}+6 p^{2}-4 p+2\right) W_{0}\right) H_{n+2}$.

(c) $(p-1)(p-2)^{2} W_{n}=\left(p W_{1}-\left(p^{2}-2 p+2\right) W_{0}\right) H_{n+2}+\left(-\left(p^{2}-2 p+2\right) W_{1}+\right.$ $\left.p\left(p^{2}-3 p+3\right) W_{0}\right) H_{n+1}$.

(d) $(p-2)^{2} W_{n}=\left(2 W_{1}-p W_{0}\right) H_{n+1}+\left(-p W_{1}+\left(p^{2}-2 p+2\right) W_{0}\right) H_{n}$.

(e) $(p-2)^{2} W_{n}=\left(2 W_{0}-2 p W_{0}+p W_{1}\right) H_{n}+(p-1)\left(-2 W_{1}+p W_{0}\right) H_{n-1}$.

(f) $(p-1)^{3}\left(W_{0}-W_{1}\right)\left(-W_{1}+(p-1) W_{0}\right) H_{n}=\left(p\left(p^{2}-3 p+3\right) W_{1}+2 W_{0}-\right.$ $\left.4 p W_{0}+3 p^{2} W_{0}-p^{3} W_{0}\right) W_{n+4}+\left(-\left(p^{4}-4 p^{3}+6 p^{2}-4 p+2\right) W_{1}+p(p-\right.$ 1) $\left.\left(p^{2}-3 p+3\right) W_{0}\right) W_{n+3}$.

(g) $(p-1)^{2}\left(W_{0}-W_{1}\right)\left(-W_{1}+(p-1) W_{0}\right) H_{n}=\left(\left(p^{2}-2 p+2\right) W_{1}-\right.$ $\left.p(p-1) W_{0}\right) W_{n+3}+\left(-p\left(p^{2}-3 p+3\right) W_{1}+(p-1)\left(p^{2}-2 p+2\right) W_{0}\right) W_{n+2}$.

(h) $(p-1)\left(W_{0}-W_{1}\right)\left(-W_{1}+(p-1) W_{0}\right) H_{n}=\left(p W_{1}-2(p-1) W_{0}\right) W_{n+2}+$ $\left(-\left(p^{2}-2 p+2\right) W_{1}+p(p-1) W_{0}\right) W_{n+1}$.

(i) $\left(W_{0}-W_{1}\right)\left(-W_{1}+(p-1) W_{0}\right) H_{n}=\left(2 W_{1}-p W_{0}\right) W_{n+1}+\left(-p W_{1}+\right.$ $\left.2(p-1) W_{0}\right) W_{n}$.

(j) $\left(W_{0}-W_{1}\right)\left(-W_{1}+(p-1) W_{0}\right) H_{n}=-\left(-p W_{1}+\left(p^{2}-2 p+2\right) W_{0}\right) W_{n}+$ $(p-1)\left(-2 W_{1}+p W_{0}\right) W_{n-1}$.

Next, we present a few basic relations between $\left\{M_{n}\right\}$ and $\left\{H_{n}\right\}$. 
Lemma 11. The following equalities are true:

$$
\begin{aligned}
(p-1)^{3} H_{n} & =p\left(p^{2}-3 p+3\right) M_{n+4}+\left(-p^{4}+4 p^{3}-6 p^{2}+4 p-2\right) M_{n+3}, \\
(p-1)^{2} H_{n} & =\left(p^{2}-2 p+2\right) M_{n+3}-p\left(p^{2}-3 p+3\right) M_{n+2}, \\
(p-1) H_{n} & =p M_{n+2}+\left(-p^{2}+2 p-2\right) M_{n+1}, \\
H_{n} & =2 M_{n+1}-p M_{n}, \\
H_{n} & =p M_{n}-2(p-1) M_{n-1},
\end{aligned}
$$

and

$$
\begin{aligned}
(p-2)^{2}(p-1)^{3} M_{n} & =p\left(p^{2}-3 p+3\right) H_{n+4}+\left(-p^{4}+4 p^{3}-6 p^{2}+4 p-2\right) H_{n+3}, \\
(p-1)^{2}(p-2)^{2} M_{n} & =\left(p^{2}-2 p+2\right) H_{n+3}-p\left(p^{2}-3 p+3\right) H_{n+2}, \\
(p-1)(p-2)^{2} M_{n} & =p H_{n+2}+\left(-p^{2}+2 p-2\right) H_{n+1}, \\
(p-2)^{2} M_{n} & =2 H_{n+1}-p H_{n}, \\
(p-2)^{2} M_{n} & =p H_{n}-2(p-1) H_{n-1} .
\end{aligned}
$$

We now present a few special identities for the generalized p-Mersenne sequence $\left\{W_{n}\right\}$.

Theorem 12. (Catalan's identity of the generalized p-Mersenne sequence) For all integers $n$ and $m$, the following identity holds:

$$
\begin{aligned}
& W_{n+m} W_{n-m}-W_{n}^{2} \\
& =\left\{\begin{array}{cc}
\frac{(p-1)^{n-m}\left((p-1)^{m}-1\right)^{2}\left(W_{0}-W_{1}\right)\left(W_{1}+(1-p) W_{0}\right)}{(p-2)^{2}} & , \text { if } p \neq 2 \\
-m^{2}\left(W_{0}-W_{1}\right)^{2} & , \text { if } p=2
\end{array} .\right.
\end{aligned}
$$

Proof. We use the identity (2.2).

As special cases of the above theorem, we have the following corollary.

Corollary 13. For all integers $n$ and $m$, the following identities hold:

(a)

$$
M_{n+m} M_{n-m}-M_{n}^{2}=\left\{\begin{array}{cc}
\frac{-(p-1)^{n-m}\left((p-1)^{m}-1\right)^{2}}{(p-2)^{2}} & , \text { if } p \neq 2 \\
-m^{2} & , \text { if } p=2
\end{array} .\right.
$$


(b)

$$
H_{n+m} H_{n-m}-H_{n}^{2}=\left\{\begin{array}{cc}
(p-1)^{n-m}\left((p-1)^{m}-1\right)^{2} & , \text { if } p \neq 2 \\
-m^{2}(p-2)^{2} & , \text { if } p=2
\end{array} .\right.
$$

Note that for $m=1$ in Catalan's identity of the generalized p-Mersenne sequence, we get the Cassini identity for the generalized p-Mersenne sequnce.

Theorem 14. (Cassini's identity of the generalized p-Mersenne sequence) For all integers $n$, the following identity holds:

$$
W_{n+1} W_{n-1}-W_{n}^{2}=\left\{\begin{array}{cc}
(p-1)^{n-1}\left(W_{0}-W_{1}\right)\left(W_{1}+(1-p) W_{0}\right) & , \text { if } p \neq 2 \\
-\left(W_{0}-W_{1}\right)^{2} & , \text { if } p=2
\end{array} .\right.
$$

As special cases of the above theorem, we have the following corollary.

Corollary 15. For all integers $n$, the following identities hold:

(a) $M_{n+1} M_{n-1}-M_{n}^{2}=\left\{\begin{array}{cc}-(p-1)^{n-1} & , \text { if } p \neq 2 \\ -1, & \text { if } p=2\end{array}\right.$

(b) $H_{n+1} H_{n-1}-H_{n}^{2}=\left\{\begin{array}{cc}(p-1)^{n-1}(p-2)^{2} & , \text { if } p \neq 2 \\ 0 & , \text { if } p=2\end{array}\right.$

The d'Ocagne's, Gelin-Cesàro's and Melham' identities can also be obtained by using 2.2. The next theorem presents d'Ocagne's, Gelin-Cesàro's and Melham' identities of generalized p-Mersenne sequence $\left\{W_{n}\right\}$.

Theorem 16. Let $n$ and $m$ be any integers. Then the following identities are true:

(a) (d'Ocagne's identity)

$$
\begin{aligned}
& W_{m+1} W_{n}-W_{m} W_{n+1} \\
& =\left\{\begin{array}{cc}
\frac{\left((p-1)^{m}-(p-1)^{n}\right)\left(W_{0}-W_{1}\right)\left((1-p) W_{0}+W_{1}\right)}{p-2} & , \text { if } p \neq 2 \\
-(m-n)\left(W_{0}-W_{1}\right)^{2} & , \text { if } p=2
\end{array} .\right.
\end{aligned}
$$


(b) (Gelin-Cesàro's identity)

$$
\begin{aligned}
W_{n+2} W_{n+1} W_{n-1} W_{n-2}-W_{n}^{4} & \\
= & \begin{array}{cc}
\frac{(p-1)^{n-3}\left(W_{0}-W_{1}\right)\left(-W_{1}+(p-1) W_{0}\right)}{(p-2)^{2}} \Omega_{1} & \text { if } p \neq 2 \\
\Omega_{2} & , \text { if } p=2
\end{array}
\end{aligned}
$$

where

$\Omega_{1}=\left(-(p-1)^{2 n}(p-1)\left(p^{2}+p-1\right)+(p-1)^{n}\left(p^{4}-2 p^{3}+4 p^{2}-4 p+2\right)-p^{3}+\right.$ $2 p-1) W_{1}^{2}+(p-1)\left(-(p-1)^{2 n}\left(p^{2}+p-1\right)+(p-1)^{n}\left(p^{4}-2 p^{3}+4 p^{2}-4 p+2\right)-\right.$ $\left.p^{4}+p^{3}+2 p^{2}-3 p+1\right) W_{0}^{2}+\left(2(p-1)^{2 n}(p-1)\left(p^{2}+p-1\right)+(p-1)^{n} p\left(-p^{4}+\right.\right.$ $\left.\left.2 p^{3}-4 p^{2}+4 p-2\right)+2 p^{4}-2 p^{3}-4 p^{2}+6 p-2\right) W_{0} W_{1}$

and

$\Omega_{2}=-\left(W_{0}-W_{1}\right)^{2}\left(\left(5 n^{2}-4\right) W_{1}^{2}+\left(5 n^{2}-10 n+1\right) W_{0}^{2}-2\left(5 n^{2}-5 n-4\right) W_{0} W_{1}\right)$.

(c) (Melham's identity)

$$
\begin{aligned}
W_{n+1} W_{n+2} W_{n+6}-W_{n+3}^{3} \\
= \begin{cases}\frac{(p-1)^{n+1}\left(W_{0}-W_{1}\right)\left(-W_{1}+(p-1) W_{0}\right)}{p-2} \Delta, & \text { if } p \neq 2 \\
\left(W_{0}-W_{1}\right)^{2}\left(-(7 n+15) W_{1}+(7 n+8) W_{0}\right) & , \text { if } p=2\end{cases}
\end{aligned}
$$

where

$$
\begin{aligned}
& \Delta=-\left((p-1)^{n}\left(p^{3}-p+1\right)(p-1)^{2}-p^{3}+p^{2}-2 p+1\right) W_{1}+(p-1)((p- \\
& \left.1)^{n+1}\left(p^{3}-p+1\right)-p^{3}+p^{2}-2 p+1\right) W_{0} .
\end{aligned}
$$

Proof. Use the identity (2.2).

As special cases of the above theorem, we have the following three corollaries. First one presents d'Ocagne's, Gelin-Cesàro's and Melham' identities of p-Mersenne sequence $\left\{M_{n}\right\}$.

Corollary 17. Let $n$ and $m$ be any integers. Then the following identities are true: 
(a) (d'Ocagne's identity)

$$
M_{m+1} M_{n}-M_{m} M_{n+1}=\left\{\begin{array}{cc}
-\frac{(p-1)^{m}-(p-1)^{n}}{p-2} & , \text { if } p \neq 2 \\
-(m-n) & , \text { if } p=2
\end{array} .\right.
$$

(b) (Gelin-Cesàro's identity)

$$
M_{n+2} M_{n+1} M_{n-1} M_{n-2}-M_{n}^{4}=\left\{\begin{array}{cc}
\frac{(p-1)^{n-3}}{(p-2)^{2}} \Psi_{1} & , \text { if } p \neq 2 \\
-\left(5 n^{2}-4\right) & , \text { if } p=2
\end{array}\right.
$$

where

$$
\Psi_{1}=-(p-1)^{2 n}(p-1)\left(p^{2}+p-1\right)+(p-1)^{n}\left(p^{4}-2 p^{3}-4 p+4 p^{2}+2\right)-p^{3}+2 p-1 .
$$

(c) (Melham's identity)

$$
M_{n+1} M_{n+2} M_{n+6}-M_{n+3}^{3}=\left\{\begin{array}{cc}
\frac{-(p-1)^{n+1}}{p-2} \Psi_{2} & , \text { if } p \neq 2 \\
-(7 n+15) & , \text { if } p=2
\end{array}\right.
$$

where

$$
\Psi_{2}=\left((p-1)^{n+2}\left(p^{3}-p+1\right)-p^{3}+p^{2}-2 p+1\right) .
$$

Second one presents d'Ocagne's, Gelin-Cesàro's and Melham's identities of p-Mersenne-Lucas sequence $\left\{H_{n}\right\}$.

Corollary 18. Let $n$ and $m$ be any integers. Then the following identities are true:

(a) (d'Ocagne's identity)

$$
H_{m+1} H_{n}-H_{m} H_{n+1}=\left\{\begin{array}{cc}
\left((p-1)^{m}-(p-1)^{n}\right)(p-2) & , \text { if } p \neq 2 \\
-(m-n)(p-2)^{2} & , \text { if } p=2
\end{array} .\right.
$$

(b) (Gelin-Cesàro's identity)

$$
H_{n+2} H_{n+1} H_{n-1} H_{n-2}-H_{n}^{4}=\left\{\begin{array}{cc}
(p-1)^{n-3}(p-2)^{2} \Gamma_{1} & , \text { if } p \neq 2 \\
(p-2)^{2} \Gamma_{2} & , \text { if } p=2
\end{array}\right.
$$


where

$\Gamma_{1}=\left((p-1)^{2 n+1}\left(p^{2}+p-1\right)+(p-1)^{n}\left(p^{4}-2 p^{3}+4 p^{2}-4 p+2\right)+p^{3}-2 p+1\right)$

and

$\Gamma_{2}=\left(-5(p-2)^{2} n^{2}-20(p-2) n+4\left(p^{2}-4 p-1\right)\right)$.

(c) (Melham's identity)

$$
H_{n+1} H_{n+2} H_{n+6}-H_{n+3}^{3}=\left\{\begin{array}{cc}
-(p-1)^{n+1}(p-2)^{2} \Gamma_{3} & , \text { if } p \neq 2 \\
(p-2)^{2} \Gamma_{4} & , \text { if } p=2
\end{array} .\right.
$$

where

$\Gamma_{3}=\left(-(p-1)^{n+2}\left(p^{3}-p+1\right)-p^{3}+p^{2}-2 p+1\right)$

and

$\Gamma_{4}=(14 n-15 p-7 n p+16)$.

\section{On the Recurrence Properties of Generalized p-Mersenne Sequence}

Taking $r=p, s=-(p-1)$ in Theorem 1 (a) and (b), we obtain the following Proposition.

Proposition 19. For $n \in \mathbb{Z}$, generalized $p$-Mersenne numbers (the case $r=p, s=$ $-(p-1))$ have the following identity:

$$
\begin{aligned}
W_{-n}= & -(p-1)^{-n}\left(W_{n}-H_{n} W_{0}\right) \\
= & \frac{-(p-1)^{-n}}{-W_{1}^{2}-(p-1) W_{0}^{2}+p W_{0} W_{1}} \\
& \times\left(\left(2 W_{1}-p W_{0}\right) W_{0} W_{n+1}-\left(W_{1}^{2}-(p-1) W_{0}^{2}\right) W_{n}\right)
\end{aligned}
$$

From the above Proposition, we have the following corollary which gives the connection between the special cases of generalized p-Mersenne sequence at the positive index and the negative index: for p-Mersenne and p-Mersenne-Lucas numbers: take $W_{n}=M_{n}$ with $M_{0}=0, M_{1}=1$ and take $W_{n}=H_{n}$ with $H_{0}=$ $2, H_{1}=p$, respectively. Note that in this case $H_{n}=H_{n}$. 
Corollary 20. For $n \in \mathbb{Z}$, we have the following recurrence relations:

(a) p-Mersenne sequence:

$$
M_{-n}=-(p-1)^{-n} M_{n}=\left\{\begin{array}{cc}
-\frac{1}{p-2}+(p-1)^{-n} \frac{1}{p-2} & , \text { if } p \neq 2 \\
-n & , \text { if } p=2
\end{array}\right.
$$

(b) p-Mersenne-Lucas sequence:

$$
H_{-n}=(p-1)^{-n} H_{n}=\left\{\begin{array}{cl}
1+(p-1)^{-n} & , \text { if } p \neq 2 \\
2 & , \text { if } p=2
\end{array}=1+(p-1)^{-n} .\right.
$$

\section{The Sum Formula $\sum_{k=0}^{n} x^{k} W_{m k+j}$}

In this section, we present sum formulas of generalized p-Mersenne numbers. The following theorem presents sum formulas of generalized p-Mersenne numbers (the case $r=p, s=-(p-1))$.

Theorem 21. Let $x$ be a real (or complex) number. For all integers $m$ and $j$, for generalized $p$-Mersenne numbers we have the following sum formulas:

(a) If $(p-1)^{m} x^{2}-x H_{m}+1 \neq 0$, then

$$
\sum_{k=0}^{n} x^{k} W_{m k+j}=\frac{\left((p-1)^{m} x-H_{m}\right) x^{n+1} W_{m n+j}+(p-1)^{m} x^{n+1} W_{m n+j-m}}{+W_{j}-(p-1)^{m} x W_{j-m}} .
$$

(b) If $(p-1)^{m} x^{2}-x H_{m}+1=u(x-a)(x-b)=0$ for some $u, a, b \in \mathbb{C}$ with $u \neq 0$ and $a \neq b$, i.e., $x=a$ or $x=b$, then

$$
\sum_{k=0}^{n} x^{k} W_{m k+j}=\frac{\begin{array}{c}
\left(x(n+2)(p-1)^{m}-(n+1) H_{m}\right) x^{n} W_{j+m n} \\
+(p-1)^{m}(n+1) x^{n} W_{m n+j-m}-(p-1)^{m} W_{j-m}
\end{array}}{2(p-1)^{m} x-H_{m}} .
$$


(c) If $(p-1)^{m} x^{2}-x H_{m}+1=u(x-c)^{2}=0$ for some $u, c \in \mathbb{C}$ with $u \neq 0$, i.e., $x=c$, then

$$
\sum_{k=0}^{n} x^{k} W_{m k+j}=\frac{(n+1)\left((p-1)^{m}(n+2) x^{n}-n x^{n-1} H_{m}\right) W_{m n+j}}{+n(n+1)(p-1)^{m} x^{n-1} W_{m n+j-m}} .
$$

Proof. Take $r=p, s=-(p-1)$ and $H_{n}=H_{n}$ in Theorem 2 .

Note that $(6.1)$ can be written in the following form

$$
\sum_{k=1}^{n} x^{k} W_{m k+j}=\frac{\begin{array}{c}
\left((p-1)^{m} x-H_{m}\right) x^{n+1} W_{m n+j}+(p-1)^{m} x^{n+1} W_{m n+j-m} \\
+x\left(H_{m}-(p-1)^{m} x\right) W_{j}-(p-1)^{m} x W_{j-m}
\end{array}}{(p-1)^{m} x^{2}-x H_{m}+1} .
$$

As special cases of $m$ and $j$ in the last Theorem, we obtain the following proposition.

Proposition 22. For generalized $p$-Mersenne numbers (the case $r=p, s=-(p-$ 1)) we have the following sum formulas:

(a) $(m=1, j=0)$

$$
\text { If }(p-1) x^{2}-p x+1 \neq 0, \text { i.e., } x \neq 1, x \neq \frac{1}{p-1} \text {, then }
$$$$
\sum_{k=0}^{n} x^{k} W_{k}=\frac{((p-1) x-p) x^{n+1} W_{n}+(p-1) x^{n+1} W_{n-1}+x W_{1}+(1-p x) W_{0}}{(p-1) x^{2}-p x+1}
$$

and

if $(p-1) x^{2}-p x+1=0$, i.e., $x=1$ or $x=\frac{1}{p-1}$, then if $p \neq 2$, then

$$
\sum_{k=0}^{n} x^{k} W_{k}=\frac{(x(n+2)(p-1)-(n+1) p) x^{n} W_{n}+(p-1)(n+1) x^{n} W_{n-1}}{+W_{1}-p W_{0}}
$$

and if $p=2$, then

$$
\sum_{k=0}^{n} x^{k} W_{k}=\frac{(n+1)\left((n+2) x^{n}-2 n x^{n-1}\right) W_{n}+n(n+1) x^{n-1} W_{n-1}}{2} .
$$


(b) $(m=2, j=0)$

If $(p-1)^{2} x^{2}-\left(p^{2}-2 p+2\right) x+1 \neq 0$, i.e., $x \neq 1, x \neq \frac{1}{p^{2}-2 p+1}$, then

$\sum_{k=0}^{n} x^{k} W_{2 k}=\frac{\left((p-1)^{2} x-\left(p^{2}-2 p+2\right)\right) x^{n+1} W_{2 n}+(p-1)^{2} x^{n+1} W_{2 n-2}}{+p x W_{1}-\left(x\left(p^{2}-p+1\right)-1\right) W_{0}}$

and

if $(p-1)^{2} x^{2}-\left(p^{2}-2 p+2\right) x+1=0$, i.e., $x=1$ or $x=\frac{1}{p^{2}-2 p+1}$, then if $p \neq 0$ and $p \neq 2$, then

$$
\sum_{k=0}^{n} x^{k} W_{2 k}=\frac{\begin{array}{c}
\left(x(n+2)(p-1)^{2}-(n+1)\left(p^{2}-2 p+2\right)\right) x^{n} W_{2 n} \\
+(p-1)^{2}(n+1) x^{n} W_{2 n-2}+p W_{1}-\left(p^{2}-p+1\right) W_{0}
\end{array}}{2(p-1)^{2} x-\left(p^{2}-2 p+2\right)}
$$

and if $p=0$ or $p=2$ (so that $(p-1)^{2}=1$ and $p^{2}-2 p+2=2$ ), then

$$
\sum_{k=0}^{n} x^{k} W_{2 k}=\frac{(n+1) x^{n-1}}{2}\left((2 x+n x-2 n) W_{2 n}+n W_{2 n-2}\right) .
$$

(c) $(m=2, j=1)$

If $(p-1)^{2} x^{2}-\left(p^{2}-2 p+2\right) x+1 \neq 0$, i.e., $x \neq 1, x \neq \frac{1}{p^{2}-2 p+1}$, then

$$
\sum_{k=0}^{n} x^{k} W_{2 k+1}=\frac{\begin{array}{c}
\left((p-1)^{2} x-\left(p^{2}-2 p+2\right)\right) x^{n+1} W_{2 n+1} \\
+(p-1)^{2} x^{n+1} W_{2 n-1}+(p x-x+1) W_{1}-p(p-1) x W_{0}
\end{array}}{(p-1)^{2} x^{2}-\left(p^{2}-2 p+2\right) x+1}
$$

and

if $(p-1)^{2} x^{2}-\left(p^{2}-2 p+2\right) x+1=0$, i.e., $x=1$ or $x=\frac{1}{p^{2}-2 p+1}$, then if $p \neq 0$ and $p \neq 2$, then

$$
\sum_{k=0}^{n} x^{k} W_{2 k+1}=\frac{\begin{array}{c}
\left(x(n+2)(p-1)^{2}-(n+1)\left(p^{2}-2 p+2\right)\right) x^{n} W_{2 n+1} \\
+(p-1)^{2}(n+1) x^{n} W_{2 n-1}+(p-1)\left(W_{1}-p W_{0}\right)
\end{array}}{2(p-1)^{2} x-\left(p^{2}-2 p+2\right)}
$$

and if $p=0$ or $p=2$ (so that $(p-1)^{2}=1$ and $p^{2}-2 p+2=2$ ), then $\sum_{k=0}^{n} x^{k} W_{2 k+1}=\frac{(n+1)\left((n+2) x^{n}-2 n x^{n-1}\right) W_{2 n+1}+n(n+1) x^{n-1} W_{2 n-1}}{2}$. 
(d) $(m=-1, j=0)$

$$
\begin{aligned}
& \text { If } \frac{(x-1)(p-x-1)}{p-1} \neq 0, \text { i.e., } x \neq 1, x \neq p-1, \text { then } \\
& \qquad \sum_{k=0}^{n} x^{k} W_{-k}=\frac{(x-p) x^{n+1} W_{-n}+x^{n+1} W_{-n+1}+(p-1) W_{0}-x W_{1}}{-(x-1)(p-x-1)}
\end{aligned}
$$

and

$$
\begin{aligned}
& \text { if } \frac{(x-1)(p-x-1)}{p-1}=0 \text {, i.e., } x=1 \text { or } x=p-1, \text { then if } p \neq 2, \text { then } \\
& \qquad \sum_{k=0}^{n} x^{k} W_{-k}=\frac{(x(n+2)-(n+1) p) x^{n} W_{-n}+(n+1) x^{n} W_{-n+1}-W_{1}}{2 x-p}
\end{aligned}
$$

and if $p=2$, then

$$
\sum_{k=0}^{n} x^{k} W_{-k}=\frac{(n+1)\left((n+2) x^{n}-2 n x^{n-1}\right) W_{-n}+n(n+1) x^{n-1} W_{-n+1}}{2} .
$$

(e) $(m=-2, j=0)$

$$
\begin{aligned}
& \text { If } \frac{(x-1)\left(-x+p^{2}-2 p+1\right)}{(p-1)^{2}} \neq 0, \text { i.e., } x \neq 1, x \neq p^{2}-2 p+1, \text { then } \\
& \sum_{k=0}^{n} x^{k} W_{-2 k}=\frac{x^{n+1} W_{-2 n+2}+\left(x-\left(p^{2}-2 p+2\right)\right) x^{n+1} W_{-2 n}}{-p x W_{1}+(p-1)(x+p-1) W_{0}} \\
& -(x-1)\left(-x+p^{2}-2 p+1\right)
\end{aligned}
$$

and

if $\frac{(x-1)\left(-x+p^{2}-2 p+1\right)}{(p-1)^{2}}=0$, i.e., $x=1$ or $x=p^{2}-2 p+1$, then if $p \neq 0$ and $p \neq 2$, then

$$
\sum_{k=0}^{n} x^{k} W_{-2 k}=\frac{(n+1) x^{n} W_{-2 n+2}+\left(x(n+2)-(n+1)\left(\begin{array}{c}
\left.\left.p^{2}-2 p+2\right)\right) x^{n} W_{-2 n} \\
-p W_{1}+(p-1) W_{0}
\end{array}\right.\right.}{2 x-\left(p^{2}-2 p+2\right)}
$$

and if $p=0$ or $p=2$ (so that $(p-1)^{2}=1$ and $p^{2}-2 p+2=2$ ), then

$\sum_{k=0}^{n} x^{k} W_{-2 k}=\frac{n(n+1) x^{n-1} W_{-2 n+2}+(n+1)\left((n+2) x^{n}-2 n x^{n-1}\right) W_{-2 n}}{2}$. 
(f) $(m=-2, j=1)$

$$
\begin{aligned}
& \text { If } \frac{(x-1)\left(-x+p^{2}-2 p+1\right)}{(p-1)^{2}} \neq 0, \text { i.e., } x \neq 1, x \neq p^{2}-2 p+1, \text { then } \\
& \sum_{k=0}^{n} x^{k} W_{-2 k+1}=\frac{x^{n+1} W_{-2 n+3}+\left(x-\left(p^{2}-2 p+2\right)\right) x^{n+1} W_{-2 n+1}+\left(-p^{2} x\right.}{\left.+p x-x+p^{2}-2 p+1\right) W_{1}+p x(p-1) W_{0}} \\
& -(x-1)\left(-x+p^{2}-2 p+1\right)
\end{aligned}
$$

and

if $\frac{(x-1)\left(-x+p^{2}-2 p+1\right)}{(p-1)^{2}}=0$, i.e., $x=1$ or $x=p^{2}-2 p+1$, then if $p \neq 0$ and $p \neq 2$, then

$\sum_{k=0}^{n} x^{k} W_{-2 k+1}$

$$
=\frac{\begin{array}{c}
(n+1) x^{n} W_{-2 n+3}+\left(x(n+2)-(n+1)\left(p^{2}-2 p+2\right)\right) x^{n} W_{-2 n+1} \\
-\left(p^{2}-p+1\right) W_{1}+p(p-1) W_{0}
\end{array}}{2 x-\left(p^{2}-2 p+2\right)}
$$

and if $p=0$ or $p=2$ (so that $(p-1)^{2}=1$ and $p^{2}-2 p+2=2$ ), then

$$
\sum_{k=0}^{n} x^{k} W_{-2 k+1}=\frac{n(n+1) x^{n-1} W_{-2 n+3}+(n+1)\left((n+2) x^{n}-2 n x^{n-1}\right) W_{-2 n+1}}{2} .
$$

From the above proposition, we have the following corollary which gives sum formulas of p-Mersenne numbers (take $W_{n}=M_{n}$ with $M_{0}=0, M_{1}=1$ ).

Corollary 23. For $n \geq 0, p$-Mersenne numbers have the following properties:

(a) $(m=1, j=0)$

$$
\begin{aligned}
& \text { If }(p-1) x^{2}-p x+1 \neq 0, \text { i.e., } x \neq 1, x \neq \frac{1}{p-1} \text {, then } \\
& \qquad \sum_{k=0}^{n} x^{k} M_{k}=\frac{((p-1) x-p) x^{n+1} M_{n}+(p-1) x^{n+1} M_{n-1}+x}{(p-1) x^{2}-p x+1}
\end{aligned}
$$

and

if $(p-1) x^{2}-p x+1=0$, i.e., $x=1$ or $x=\frac{1}{p-1}$, then if $p \neq 2$, then

$\sum_{k=0}^{n} x^{k} M_{k}=\frac{(x(n+2)(p-1)-(n+1) p) x^{n} M_{n}+(p-1)(n+1) x^{n} M_{n-1}+1}{2(p-1) x-p}$ 
and if $p=2$, then

$$
\sum_{k=0}^{n} x^{k} M_{k}=\frac{(n+1)\left((n+2) x^{n}-2 n x^{n-1}\right) M_{n}+n(n+1) x^{n-1} M_{n-1}}{2} .
$$

(b) $(m=2, j=0)$

If $(p-1)^{2} x^{2}-\left(p^{2}-2 p+2\right) x+1 \neq 0$, i.e., $x \neq 1, x \neq \frac{1}{p^{2}-2 p+1}$, then

$\sum_{k=0}^{n} x^{k} M_{2 k}=\frac{\left((p-1)^{2} x-\left(p^{2}-2 p+2\right)\right) x^{n+1} M_{2 n}+(p-1)^{2} x^{n+1} M_{2 n-2}+p x}{(p-1)^{2} x^{2}-\left(p^{2}-2 p+2\right) x+1}$

and

if $(p-1)^{2} x^{2}-\left(p^{2}-2 p+2\right) x+1=0$, i.e., $x=1$ or $x=\frac{1}{p^{2}-2 p+1}$, then if $p \neq 0$ and $p \neq 2$, then

$$
\begin{aligned}
& \left(x(n+2)(p-1)^{2}-(n+1)\left(p^{2}-2 p+2\right)\right) x^{n} M_{2 n} \\
& \sum_{k=0}^{n} x^{k} M_{2 k}=\frac{+(p-1)^{2}(n+1) x^{n} M_{2 n-2}+p}{2(p-1)^{2} x-\left(p^{2}-2 p+2\right)}
\end{aligned}
$$

and if $p=0$ or $p=2$, then

$$
\sum_{k=0}^{n} x^{k} M_{2 k}=\frac{(n+1) x^{n-1}}{2}\left((2 x+n x-2 n) M_{2 n}+n M_{2 n-2}\right) .
$$

(c) $(m=2, j=1)$

If $(p-1)^{2} x^{2}-\left(p^{2}-2 p+2\right) x+1 \neq 0$, i.e., $x \neq 1, x \neq \frac{1}{p^{2}-2 p+1}$, then

$$
\sum_{k=0}^{n} x^{k} M_{2 k+1}=\frac{\begin{array}{c}
\left((p-1)^{2} x-\left(p^{2}-2 p+2\right)\right) x^{n+1} M_{2 n+1} \\
+(p-1)^{2} x^{n+1} M_{2 n-1}+(p x-x+1)
\end{array}}{(p-1)^{2} x^{2}-\left(p^{2}-2 p+2\right) x+1}
$$

and

if $(p-1)^{2} x^{2}-\left(p^{2}-2 p+2\right) x+1=0$, i.e., $x=1$ or $x=\frac{1}{p^{2}-2 p+1}$, then if $p \neq 0$ and $p \neq 2$, then

$$
\begin{aligned}
& \left(x(n+2)(p-1)^{2}-(n+1)\left(p^{2}-2 p+2\right)\right) x^{n} M_{2 n+1} \\
& \sum_{k=0}^{n} x^{k} M_{2 k+1}=\frac{+(p-1)^{2}(n+1) x^{n} M_{2 n-1}+p-1}{2(p-1)^{2} x-\left(p^{2}-2 p+2\right)}
\end{aligned}
$$


and if $p=0$ or $p=2$, then

$$
\sum_{k=0}^{n} x^{k} M_{2 k+1}=\frac{(n+1)\left((n+2) x^{n}-2 n x^{n-1}\right) M_{2 n+1}+n(n+1) x^{n-1} M_{2 n-1}}{2} .
$$

(d) $(m=-1, j=0)$

If $\frac{(x-1)(p-x-1)}{p-1} \neq 0$, i.e., $x \neq 1, x \neq p-1$, then

$$
\sum_{k=0}^{n} x^{k} M_{-k}=\frac{(x-p) x^{n+1} M_{-n}+x^{n+1} M_{-n+1}-x}{-(x-1)(p-x-1)}
$$

and

$$
\begin{aligned}
& \text { if } \frac{(x-1)(p-x-1)}{p-1}=0, \text { i.e., } x=1 \text { or } x=p-1, \text { then if } p \neq 2, \text { then } \\
& \qquad \sum_{k=0}^{n} x^{k} M_{-k}=\frac{(x(n+2)-(n+1) p) x^{n} M_{-n}+(n+1) x^{n} M_{-n+1}-1}{2 x-p}
\end{aligned}
$$

and if $p=2$, then

$$
\sum_{k=0}^{n} x^{k} M_{-k}=\frac{(n+1)\left((n+2) x^{n}-2 n x^{n-1}\right) M_{-n}+n(n+1) x^{n-1} M_{-n+1}}{2} .
$$

(e) $(m=-2, j=0)$

$$
\begin{gathered}
\text { If } \frac{(x-1)\left(-x+p^{2}-2 p+1\right)}{(p-1)^{2}} \neq 0, \text { i.e., } x \neq 1, x \neq p^{2}-2 p+1, \text { then } \\
\sum_{k=0}^{n} x^{k} M_{-2 k}=\frac{x^{n+1} M_{-2 n+2}+\left(x-\left(p^{2}-2 p+2\right)\right) x^{n+1} M_{-2 n}-p x}{-(x-1)\left(-x+p^{2}-2 p+1\right)}
\end{gathered}
$$

and

if $\frac{(x-1)\left(-x+p^{2}-2 p+1\right)}{(p-1)^{2}}=0$, i.e., $x=1$ or $x=p^{2}-2 p+1$, then if $p \neq 0$ and $p \neq 2$, then

$$
\sum_{k=0}^{n} x^{k} M_{-2 k}=\frac{(n+1) x^{n} M_{-2 n+2}+\left(x(n+2)-(n+1)\left(p^{2}-2 p+2\right)\right) x^{n} M_{-2 n}-p}{2 x-\left(p^{2}-2 p+2\right)}
$$


and if $p=0$ or $p=2$, then

$$
\sum_{k=0}^{n} x^{k} M_{-2 k}=\frac{n(n+1) x^{n-1} M_{-2 n+2}+(n+1)\left((n+2) x^{n}-2 n x^{n-1}\right) M_{-2 n}}{2} .
$$

(f) $(m=-2, j=1)$

$$
\begin{aligned}
& \text { If } \frac{(x-1)\left(-x+p^{2}-2 p+1\right)}{(p-1)^{2}} \neq 0, \text { i.e., } x \neq 1, x \neq p^{2}-2 p+1, \text { then } \\
& \sum_{k=0}^{n} x^{k} M_{-2 k+1}=\frac{x^{n+1} M_{-2 n+3}+\left(x-\left(p^{2}-2 p+2\right)\right) x^{n+1} M_{-2 n+1}}{+\left(-p^{2} x+p x-x+p^{2}-2 p+1\right)} \\
& -(x-1)\left(-x+p^{2}-2 p+1\right)
\end{aligned}
$$

and

if $\frac{(x-1)\left(-x+p^{2}-2 p+1\right)}{(p-1)^{2}}=0$, i.e., $x=1$ or $x=p^{2}-2 p+1$, then if $p \neq 0$ and $p \neq 2$, then

$$
\sum_{k=0}^{n} x^{k} M_{-2 k+1}=\frac{\begin{array}{c}
(n+1) x^{n} M_{-2 n+3}+(x(n+2) \\
\left.-(n+1)\left(p^{2}-2 p+2\right)\right) x^{n} M_{-2 n+1}-\left(p^{2}-p+1\right)
\end{array}}{2 x-\left(p^{2}-2 p+2\right)}
$$

and if $p=0$ or $p=2$, then

$$
\sum_{k=0}^{n} x^{k} M_{-2 k+1}=\frac{n(n+1) x^{n-1} M_{-2 n+3}+(n+1)\left((n+2) x^{n}-2 n x^{n-1}\right) M_{-2 n+1}}{2} .
$$

Taking $W_{n}=H_{n}$ with $H_{0}=2, H_{1}=p$ in the last proposition, we have the following corollary which presents sum formulas of p-Mersenne-Lucas numbers.

Corollary 24. For $n \geq 0$, p-Mersenne-Lucas numbers have the following properties:

(a) $(m=1, j=0)$

$$
\begin{aligned}
& \text { If }(p-1) x^{2}-p x+1 \neq 0, \text { i.e., } x \neq 1, x \neq \frac{1}{p-1}, \text { then } \\
& \qquad \sum_{k=0}^{n} x^{k} H_{k}=\frac{((p-1) x-p) x^{n+1} H_{n}+(p-1) x^{n+1} H_{n-1}+2-p x}{(p-1) x^{2}-p x+1}
\end{aligned}
$$


and

if $(p-1) x^{2}-p x+1=0$, i.e., $x=1$ or $x=\frac{1}{p-1}$, then if $p \neq 2$, then

$\sum_{k=0}^{n} x^{k} H_{k}=\frac{(x(n+2)(p-1)-(n+1) p) x^{n} H_{n}+(p-1)(n+1) x^{n} H_{n-1}-p}{2(p-1) x-p}$

and if $p=2$ then

$$
\sum_{k=0}^{n} x^{k} H_{k}=\frac{(n+1)\left((n+2) x^{n}-2 n x^{n-1}\right) H_{n}+n(n+1) x^{n-1} H_{n-1}}{2} .
$$

(b) $(m=2, j=0)$

If $(p-1)^{2} x^{2}-\left(p^{2}-2 p+2\right) x+1 \neq 0$, i.e., $x \neq 1, x \neq \frac{1}{p^{2}-2 p+1}$, then

$\sum_{k=0}^{n} x^{k} H_{2 k}=\frac{\left((p-1)^{2} x-\left(p^{2}-2 p+2\right)\right) x^{n+1} H_{2 n}+(p-1)^{2} x^{n+1} H_{2 n-2}}{-x\left(p^{2}-2 p+2\right)+2}$

and

if $(p-1)^{2} x^{2}-\left(p^{2}-2 p+2\right) x+1=0$, i.e., $x=1$ or $x=\frac{1}{p^{2}-2 p+1}$, then if $p \neq 0$ and $p \neq 2$, then

$$
\sum_{k=0}^{n} x^{k} H_{2 k}=\frac{\begin{array}{c}
\left(x(n+2)(p-1)^{2}-(n+1)\left(p^{2}-2 p+2\right)\right) x^{n} H_{2 n} \\
+(p-1)^{2}(n+1) x^{n} H_{2 n-2}-p^{2}+2 p-2
\end{array}}{2(p-1)^{2} x-\left(p^{2}-2 p+2\right)}
$$

and if $p=0$ or $p=2$, then

$$
\sum_{k=0}^{n} x^{k} H_{2 k}=\frac{(n+1) x^{n-1}}{2}\left((2 x+n x-2 n) H_{2 n}+n H_{2 n-2}\right) .
$$

(c) $(m=2, j=1)$

If $(p-1)^{2} x^{2}-\left(p^{2}-2 p+2\right) x+1 \neq 0$, i.e., $x \neq 1, x \neq \frac{1}{p^{2}-2 p+1}$, then

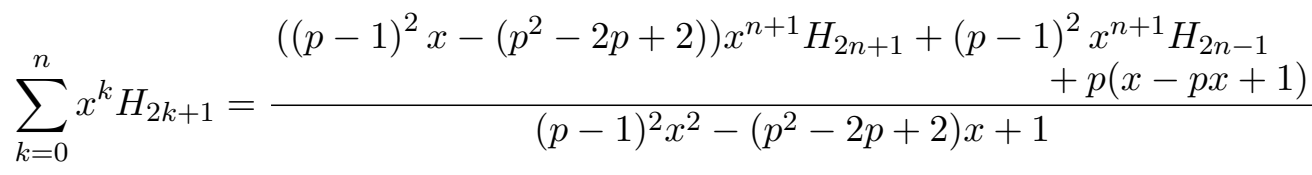


and

if $(p-1)^{2} x^{2}-\left(p^{2}-2 p+2\right) x+1=0$, i.e., $x=1$ or $x=\frac{1}{p^{2}-2 p+1}$, then if $p \neq 0$ and $p \neq 2$, then

$$
\sum_{k=0}^{n} x^{k} H_{2 k+1}=\frac{\begin{array}{c}
\left(x(n+2)(p-1)^{2}-(n+1)\left(p^{2}-2 p+2\right)\right) x^{n} H_{2 n+1} \\
+(p-1)^{2}(n+1) x^{n} H_{2 n-1}-p(p-1)
\end{array}}{2(p-1)^{2} x-\left(p^{2}-2 p+2\right)}
$$

and if $p=0$ or $p=2$, then

$\sum_{k=0}^{n} x^{k} H_{2 k+1}=\frac{(n+1)\left((n+2) x^{n}-2 n x^{n-1}\right) H_{2 n+1}+n(n+1) x^{n-1} H_{2 n-1}}{2}$.

(d) $(m=-1, j=0)$

If $\frac{(x-1)(p-x-1)}{p-1} \neq 0$, i.e., $x \neq 1, x \neq p-1$, then

$$
\sum_{k=0}^{n} x^{k} H_{-k}=\frac{(x-p) x^{n+1} H_{-n}+x^{n+1} H_{-n+1}-p x+2 p-2}{-(x-1)(p-x-1)}
$$

and

if $\frac{(x-1)(p-x-1)}{p-1}=0$, i.e., $x=1$ or $x=p-1$, then if $p \neq 2$, then

$$
\sum_{k=0}^{n} x^{k} H_{-k}=\frac{(x(n+2)-(n+1) p) x^{n} H_{-n}+(n+1) x^{n} H_{-n+1}-p}{2 x-p}
$$

and if $p=2$, then

$$
\sum_{k=0}^{n} x^{k} H_{-k}=\frac{(n+1)\left((n+2) x^{n}-2 n x^{n-1}\right) H_{-n}+n(n+1) x^{n-1} H_{-n+1}}{2} .
$$

(e) $(m=-2, j=0)$

$$
\begin{array}{r}
\text { If } \frac{(x-1)\left(-x+p^{2}-2 p+1\right)}{(p-1)^{2}} \neq 0, \text { i.e., } x \neq 1, x \neq p^{2}-2 p+1, \text { then } \\
\sum_{k=0}^{n} x^{k} H_{-2 k}=\frac{x^{n+1} H_{-2 n+2}+\left(x-\left(p^{2}-2 p+2\right)\right) x^{n+1} H_{-2 n}}{-(x-1)\left(-x+p^{2}-2 p+1\right)}
\end{array}
$$


and

if $\frac{(x-1)\left(-x+p^{2}-2 p+1\right)}{(p-1)^{2}}=0$, i.e., $x=1$ or $x=p^{2}-2 p+1$, then if $p \neq 0$ and $p \neq 2$, then

$$
\sum_{k=0}^{n} x^{k} H_{-2 k}=\frac{(n+1) x^{n} H_{-2 n+2}+\left(x(n+2)-(n+1)\left(p^{2}-2 p+2\right)\right)}{2 x-\left(p^{2}-2 p+2\right)}
$$

and if $p=0$ or $p=2$, then

$$
\sum_{k=0}^{n} x^{k} H_{-2 k}=\frac{n(n+1) x^{n-1} H_{-2 n+2}+(n+1)\left((n+2) x^{n}-2 n x^{n-1}\right) H_{-2 n}}{2} .
$$

(f) $(m=-2, j=1)$

$$
\begin{aligned}
& \text { If } \frac{(x-1)\left(-x+p^{2}-2 p+1\right)}{(p-1)^{2}} \neq 0, \text { i.e., } x \neq 1, x \neq p^{2}-2 p+1, \text { then } \\
& \sum_{k=0}^{n} x^{k} H_{-2 k+1}=\frac{x^{n+1} H_{-2 n+3}+\left(x-\left(p^{2}-2 p+2\right)\right) x^{n+1} H_{-2 n+1}}{-(x-1)\left(-x+p^{2}-2 p+1\right)}
\end{aligned}
$$

and

if $\frac{(x-1)\left(-x+p^{2}-2 p+1\right)}{(p-1)^{2}}=0$, i.e., $x=1$ or $x=p^{2}-2 p+1$, then if $p \neq 0$ and $p \neq 2$, then

$$
\sum_{k=0}^{n} x^{k} H_{-2 k+1}=\frac{\begin{array}{c}
(n+1) x^{n} H_{-2 n+3}+(x(n+2) \\
\left.-(n+1)\left(p^{2}-2 p+2\right)\right) x^{n} H_{-2 n+1}-p\left(p^{2}-3 p+3\right)
\end{array}}{2 x-\left(p^{2}-2 p+2\right)}
$$

and if $p=0$ or $p=2$, then

$$
\sum_{k=0}^{n} x^{k} H_{-2 k+1}=\frac{n(n+1) x^{n-1} H_{-2 n+3}+(n+1)\left((n+2) x^{n}-2 n x^{n-1}\right) H_{-2 n+1}}{2} .
$$

Taking $x=1$ in the last two corollaries we get the following corollaries. 
Corollary 25. Let $x=1$. For $n \geq 0, p$-Mersenne numbers have the following properties:

(a) If $p \neq 2$, then

$$
\sum_{k=0}^{n} M_{k}=\frac{1}{p-2}\left((p-n-2) M_{n}+(p-1)(n+1) M_{n-1}+1\right)
$$

and if $p=2$, then

$$
\sum_{k=0}^{n} M_{k}=\frac{1}{2}\left(-(n-2)(n+1) M_{n}+n(n+1) M_{n-1}\right) .
$$

(b) If $p \neq 0$ and $p \neq 2$, then

$$
\sum_{k=0}^{n} M_{2 k}=\frac{1}{p(p-2)}\left(\left(p^{2}-2 p-n\right) M_{2 n}+(p-1)^{2}(n+1) M_{2 n-2}+p\right)
$$

and if $p=0$ or $p=2$, then

$$
\sum_{k=0}^{n} M_{2 k}=\frac{(n+1)}{2}\left((2-n) M_{2 n}+n M_{2 n-2}\right) .
$$

(c) If $p \neq 0$ and $p \neq 2$, then

$\sum_{k=0}^{n} M_{2 k+1}=\frac{1}{p(p-2)}\left(\left(p^{2}-2 p-n\right) M_{2 n+1}+(p-1)^{2}(n+1) M_{2 n-1}+p-1\right)$

and if $p=0$ or $p=2$, then

$$
\sum_{k=0}^{n} M_{2 k+1}=\frac{1}{2}\left(-(n+1)(n-2) M_{2 n+1}+n(n+1) M_{2 n-1}\right) .
$$

(d) If $p \neq 2$, then

$$
\sum_{k=0}^{n} M_{-k}=\frac{1}{(p-2)}\left((n p-n+p-2) M_{-n}-(n+1) M_{-n+1}+1\right)
$$

and if $p=2$, then

$$
\sum_{k=0}^{n} M_{-k}=\frac{1}{2}\left(-(n+1)(n-2) M_{-n}+n(n+1) M_{-n+1}\right) .
$$


(e) If $p \neq 0$ and $p \neq 2$, then

$$
\sum_{k=0}^{n} M_{-2 k}=\frac{1}{p(p-2)}\left(-(n+1) M_{-2 n+2}+\left(n(p-1)^{2}+p(p-2)\right) M_{-2 n}+p\right)
$$

and if $p=0$ or $p=2$, then

$$
\sum_{k=0}^{n} M_{-2 k}=\frac{1}{2}\left(n(n+1) M_{-2 n+2}-(n-2)(n+1) M_{-2 n}\right) .
$$

(f) If $p \neq 0$ and $p \neq 2$, then

$$
\begin{aligned}
& \sum_{k=0}^{n} M_{-2 k+1} \\
& =\frac{1}{p(p-2)}\left(-(n+1) M_{-2 n+3}+\left(n(p-1)^{2}+p(p-2)\right) M_{-2 n+1}+\left(p^{2}-p+1\right)\right)
\end{aligned}
$$

and if $p=0$ or $p=2$, then

$$
\sum_{k=0}^{n} M_{-2 k+1}=\frac{1}{2}\left(n(n+1) M_{-2 n+3}-(n-2)(n+1) M_{-2 n+1}\right) .
$$

p-Mersenne-Lucas numbers have the following properties.

Corollary 26. Let $x=1$. For $n \geq 0$, -Mersenne-Lucas numbers have the following properties:

(a) If $p \neq 2$, then

$$
\sum_{k=0}^{n} H_{k}=\frac{1}{p-2}\left((p-n-2) H_{n}+(p-1)(n+1) H_{n-1}-p\right)
$$

and if $p=2$, then

$$
\sum_{k=0}^{n} H_{k}=\frac{1}{2}\left(-(n-2)(n+1) H_{n}+n(n+1) H_{n-1}\right) .
$$

(b) If $p \neq 0$ and $p \neq 2$, then

$$
\sum_{k=0}^{n} H_{2 k}=\frac{1}{p(p-2)}\left(\left(p^{2}-2 p-n\right) H_{2 n}+(p-1)^{2}(n+1) H_{2 n-2}-p^{2}+2 p-2\right)
$$


and if $p=0$ or $p=2$, then

$$
\sum_{k=0}^{n} H_{2 k}=\frac{(n+1)}{2}\left((2-n) H_{2 n}+n H_{2 n-2}\right) .
$$

(c) If $p \neq 0$ and $p \neq 2$, then

$$
\sum_{k=0}^{n} H_{2 k+1}=\frac{1}{p(p-2)}\left(\left(p^{2}-2 p-n\right) H_{2 n+1}+(p-1)^{2}(n+1) H_{2 n-1}-p(p-1)\right)
$$

and if $p=0$ or $p=2$, then

$$
\sum_{k=0}^{n} H_{2 k+1}=\frac{1}{2}\left(-(n-2)(n+1) H_{2 n+1}+n(n+1) H_{2 n-1}\right) .
$$

(d) If $p \neq 2$, then

$$
\sum_{k=0}^{n} H_{-k}=\frac{1}{(p-2)}\left((n(p-1)+p-2) H_{-n}-(n+1) H_{-n+1}+p\right)
$$

and if $p=2$, then

$$
\sum_{k=0}^{n} H_{-k}=\frac{1}{2}\left(-(n-2)(n+1) H_{-n}+n(n+1) H_{-n+1}\right) .
$$

(e) If $p \neq 0$ and $p \neq 2$, then

$$
\sum_{k=0}^{n} H_{-2 k}=\frac{1}{p(p-2)}\left(-(n+1) H_{-2 n+2}+\left(n(p-1)^{2}+p(p-2)\right) H_{-2 n}+p^{2}-2 p+2\right)
$$

and if $p=0$ or $p=2$, then

$$
\sum_{k=0}^{n} H_{-2 k}=\frac{1}{2}\left(n(n+1) H_{-2 n+2}-(n-2)(n+1) H_{-2 n}\right) .
$$

(f) If $p \neq 0$ and $p \neq 2$, then

$$
\begin{aligned}
& \sum_{k=0}^{n} H_{-2 k+1} \\
& =\frac{1}{p(p-2)}\left(-(n+1) H_{-2 n+3}+\left(n(p-1)^{2}+p(p-2)\right) H_{-2 n+1}+p\left(p^{2}-3 p+3\right)\right)
\end{aligned}
$$

and if $p=0$ or $p=2$, then

$$
\sum_{k=0}^{n} H_{-2 k+1}=\frac{1}{2}\left(n(n+1) H_{-2 n+3}-(n-2)(n+1) H_{-2 n+1}\right) .
$$




\section{Matrices Related with Generalized p-Mersenne Numbers}

We define the square matrix $A$ of order 2 as:

$$
A=\left(\begin{array}{cc}
p & -(p-1) \\
1 & 0
\end{array}\right)
$$

such that $\operatorname{det} A=p-1$. Then, we have

$$
\left(\begin{array}{c}
W_{n+1} \\
W_{n}
\end{array}\right)=\left(\begin{array}{cc}
p & -(p-1) \\
1 & 0
\end{array}\right)\left(\begin{array}{c}
W_{n} \\
W_{n-1}
\end{array}\right)
$$

and

$$
\left(\begin{array}{c}
W_{n+1} \\
W_{n}
\end{array}\right)=\left(\begin{array}{cc}
p & -(p-1) \\
1 & 0
\end{array}\right)^{n}\left(\begin{array}{l}
W_{1} \\
W_{0}
\end{array}\right) .
$$

If we take $W_{n}=M_{n}$ in 7.1 we have

$$
\left(\begin{array}{c}
M_{n+1} \\
M_{n}
\end{array}\right)=\left(\begin{array}{cc}
p & -(p-1) \\
1 & 0
\end{array}\right)\left(\begin{array}{c}
M_{n} \\
M_{n-1}
\end{array}\right) .
$$

We also define

$$
M_{n}=\left(\begin{array}{cc}
M_{n+1} & -(p-1) M_{n} \\
M_{n} & -(p-1) M_{n-1}
\end{array}\right)
$$

and

$$
C_{n}=\left(\begin{array}{cc}
W_{n+1} & -(p-1) W_{n} \\
W_{n} & -(p-1) W_{n-1}
\end{array}\right) .
$$

Theorem 27. For all integers $m, n$, we have

(a) $M_{n}=A^{n}$

(b) $C_{1} A^{n}=A^{n} C_{1}$

(c) $C_{n+m}=C_{n} M_{m}=M_{m} C_{n}$.

Proof. Take $r=p, s=-(p-1)$ in Soykan [25, Theorem 5.1]. 
Corollary 28. For all integers $n$, we have the following formulas for the p-Mersenne and p-Mersenne-Lucas numbers.

(a) p-Mersenne Numbers.

$$
A^{n}=\left(\begin{array}{cc}
p & -(p-1) \\
1 & 0
\end{array}\right)^{n}=\left(\begin{array}{cc}
M_{n+1} & -(p-1) M_{n} \\
M_{n} & -(p-1) M_{n-1}
\end{array}\right) .
$$

(b) $p$-Mersenne-Lucas Numbers. If $p \neq 2$, then

$$
\begin{aligned}
& A^{n}=\left(\begin{array}{cc}
p & -(p-1) \\
1 & 0
\end{array}\right)^{n}=\frac{1}{(p-2)^{2}}\left(\begin{array}{cc}
2 H_{n+2}-p H_{n+1} & -(p-1)\left(2 H_{n+1}-p H_{n}\right) \\
2 H_{n+1}-p H_{n} & -(p-1)\left(2 H_{n}-p H_{n-1}\right)
\end{array}\right) \\
& \text { and if } \left.p=2 \text { (so that } M_{n}=n, H_{n}=2\right) \text {, then } \\
& A^{n}=\left(\begin{array}{cc}
2 & -1 \\
1 & 0
\end{array}\right)^{n}=\left(\begin{array}{cc}
\frac{n+1}{2} H_{n+1} & -\frac{n}{2} H_{n} \\
\frac{n}{2} H_{n} & -\frac{n-1}{2} H_{n-1}
\end{array}\right)=\left(\begin{array}{cc}
n+1 & -n \\
n & -(n-1)
\end{array}\right) .
\end{aligned}
$$

Proof. (a) It is given in Theorem 27 (a).

(b) Note that if $p=2$, then $M_{n}=n, H_{n}=2$ and so

$$
M_{n}=n=\frac{n}{2} \times 2=\frac{n}{2} H_{n} .
$$

Note also that, from Lemma 11, we have

$$
(p-2)^{2} M_{n}=2 H_{n+1}-p H_{n}
$$

Using (7.3), (7.4) and (a), we get required result.

Theorem 29. For all integers $m, n$, we have

$$
W_{n+m}=W_{n} M_{m+1}-(p-1) W_{n-1} M_{m}
$$

Proof. Take $r=p, s=-(p-1)$ in Soykan [25, Theorem 5.2.]. 
By Lemma 9, we know that

$$
\left(W_{0}-W_{1}\right)\left(-W_{1}+(p-1) W_{0}\right) M_{n}=-W_{0} W_{n+1}+W_{1} W_{n},
$$

so 7.5 can be written in the following form

$$
W_{n+m}=\frac{\begin{array}{c}
W_{n}\left(W_{m+1}\left(W_{1}-p W_{0}\right)+W_{m} W_{0}(p-1)\right) \\
+W_{n-1}(p-1)\left(W_{0} W_{m+1}-W_{1} W_{m}\right)
\end{array}}{\left(W_{0}-W_{1}\right)\left(-W_{1}+(p-1) W_{0}\right)} .
$$

Corollary 30. For all integers $m, n$, we have

$$
\begin{aligned}
M_{n+m} & =M_{n} M_{m+1}-(p-1) M_{n-1} M_{m}, \\
H_{n+m} & =H_{n} M_{m+1}-(p-1) H_{n-1} M_{m},
\end{aligned}
$$

and (if $p \neq 2$, then)

$$
H_{n+m}=\frac{H_{n}\left(-p H_{m+1}+2(p-1) H_{m}\right)+H_{n-1}(p-1)\left(2 H_{m+1}-p H_{m}\right)}{(p-2)^{2}} .
$$

Note that if $p=2$ (so that for all integers $n, H_{n}=2$ ), then for all integers $m, n, H_{n+m}=2$.

\section{References}

[1] J. J. Bravo and C. A. Gómez, Mersenne k-Fibonacci numbers, Glas. Mat. Ser. III 51(2) (2016), 307-319. https://doi.org/10.3336/gm.51.2.02

[2] J. Brillhart, On the factors of certain Mersenne numbers, Math. Comp. 14(72) (1960), 365-369. https://doi.org/10.1090/S0025-5718-1960-0123507-6

[3] J. Brillhart, On the factors of certain Mersenne numbers, II, Math. Comp. 18 (1964), 87-92. https://doi.org/10.1090/S0025-5718-1964-0159776-X

[4] P. Catarino, H. Campos and P. Vasco, On the Mersenne sequence, Ann. Math. Inform. 46 (2016), 37-53.

[5] M. Chelgham and A. Boussayoud, On the $k$-Mersenne-Lucas numbers, Notes on Number Theory and Discrete Mathematics 27(1) (2021), 7-13.

https://doi.org/10.7546/nntdm.2021.27.1.7-13 
[6] A. Daşdemir, Mersene, Jacobsthal, and Jacobsthal-Lucas numbers with negative subscripts, Acta Math. Univ. Comenian. (N.S.) 88(1) (2019), 145-156.

[7] J. R. Ehrman, The number of prime divisors of certain Mersenne numbers, Math. Comp. 21(100) (1967), 700-704. https://doi.org/10.1090/S0025-5718-1967-0223320-1

[8] K. Ford, F. Luca and I. E. Shparlinski, On the largest prime factor of the Mersenne numbers, Bull. Austr. Math. Soc. 79(3) (2009), 455-463. https://doi.org/10.1017/S0004972709000033

[9] T. Goy, On new identities for Mersenne numbers, Appl. Math. E-Notes 18 (2018), 100-105.

[10] R. Granger and A. Moss, Generalized Mersenne numbers revisited, Math. Comp. 82(284) (2013), 2389-2420. https://doi.org/10.1090/S0025-5718-2013-02704-4

[11] A. F. Horadam, A generalized Fibonacci sequence, Amer. Math. Monthly 68 (1961), 455-459. https://doi.org/10.1080/00029890.1961.11989696

[12] A. F. Horadam, Basic properties of a certain generalized sequence of numbers, Fibonacci Quarterly 3.3 (1965), 161-176.

[13] A. F. Horadam, Special properties of the sequence $w_{n}(a, b ; p, q)$, Fibonacci Quarterly 5(5) (1967), 424-434.

[14] A. F. Horadam, Generating functions for powers of a certain generalized sequence of numbers, Duke Math. J. 32 (1965), 437-446.

https://doi.org/10.1215/S0012-7094-65-03244-8

[15] J. H. Jaroma and K. N. Reddy, Classical and alternative approaches to the Mersenne and Fermat numbers, Amer. Math. Monthly 114(8) (2007), 677-687. https://doi.org/10.1080/00029890.2007.11920459

[16] L. Murata and C. Pomerance, On the largest prime factor of a Mersenne number, Number Theory 36 (2004), 209-218. https://doi.org/10.1090/crmp/036/16

[17] P. Ochalik and A. Włoch, On generalized Mersenne numbers, their interpretations and matrix generators, Ann. Univ. Mariae Curie-Sktodowska Sect. A 72(1) (2018), 69-76. https://doi.org/10.17951/a.2018.72.1.69-76 
[18] C. Pomerance, On primitive divisors of Mersenne numbers, Acta Arith. 46 (1986), 355-367. https://doi.org/10.4064/aa-46-4-355-367

[19] Samuel S. Wagstaff, Divisors of Mersenne numbers, Math. Comp. 40 (1983), 385-397. https://doi.org/10.1090/S0025-5718-1983-0679454-X

[20] A. Schinzel, On primitive prime factors of $a^{n}-b^{n}$, Proc. Cambridge Philos. Soc. 58(4) (1962), 555-562. https://doi.org/10.1017/S0305004100040561

[21] N. J. A. Sloane, The on-line encyclopedia of integer sequences, http://oeis.org/

[22] J. A. Solinas, Generalized Mersenne numbers, Technical report CORR-39, Dept. of C\&O, University of Waterloo, 1999. Available from http://www.cacr.math.uwaterloo.ca

[23] Y. Soykan, A study on generalized Mersenne numbers, Journal of Progressive Research in Mathematics 18(3) (2021), 90-112.

[24] Y. Soykan, Simson identity of generalized $m$-step Fibonacci numbers, Int. J. Adv. Appl. Math. Mech. 7(2)( 2019), 45-56.

[25] Y. Soykan, On generalized $(r, s)$-numbers, International Journal of Advances in Applied Mathematics and Mechanics 8(1) (2020), 1-14.

[26] Y. Soykan, Some properties of generalized Fibonacci numbers: identities, recurrence properties and closed forms of the sum formulas $\sum_{k=0}^{n} x^{k} W_{m k+j}$, Archives of Current Research International 21(3) (2021), 11-38.

https://doi.org/10.9734/acri/2021/v21i330235

[27] C. L. Stewart, The greatest prime factor of $a^{n}-b^{n}$, Acta Arith. 26 (1975), 427-433. https://doi.org/10.4064/aa-26-4-427-433

[28] R. Zatorsky and T. Goy, Parapermanents of triangular matrices and some general theorems on number sequences, J. Integer Seq. 19 (2016), Article 16.2.2.

This is an open access article distributed under the terms of the Creative Commons Attribution License (http://creativecommons.org/licenses/by/4.0/), which permits unrestricted, use, distribution and reproduction in any medium, or format for any purpose, even commercially provided the work is properly cited. 